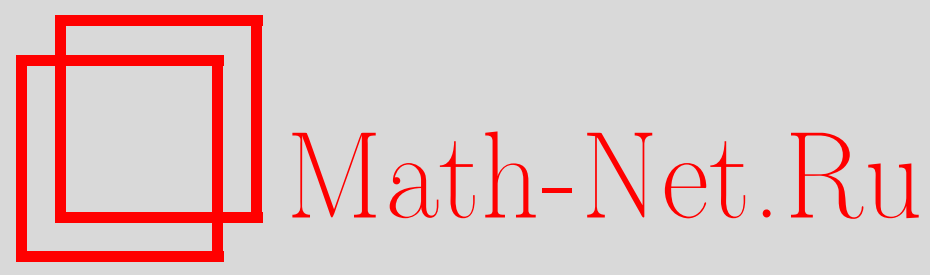

С. В. Асташкин, О выделении подсистем, "мажорируемых" системой Радемахера, Матем. заметки, 1999, том 65, выпуск 4, 483-495

DOI: https://doi.org/10.4213/mzm1075

Использование Общероссийского математического портала Math-Net.Ru подразумевает, что вы прочитали и согласны с пользовательским соглашением http://www . mathnet.ru/rus/agreement

Параметры загрузки:

IP : 54.162 .27 .143

26 апреля 2023 г., 18:20:42

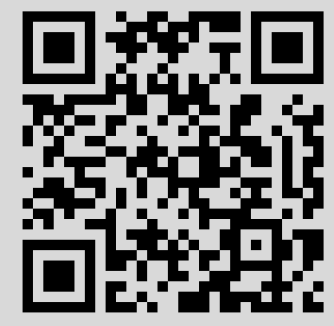


УДК 517.982 .27

\section{О ВЫДЕЛЕНИИ ПОДСИСТЕМ, “МАЖОРИРУЕМЫХ" СИСТЕМОЙ РАДЕМАХЕРА}

\section{С. В. Асташкин}

В работе доказано, что из любой равномерно ограниченной ортонормированной системы $\left\{f_{n}\right\}_{n=1}^{\infty}$ случайных величин, определенных на вероятностном пространстве $(\Omega, \Sigma, \mathrm{P})$, можно выделить подсистему $\left\{f_{n_{i}}\right\}_{i=1}^{\infty}$, мажорируемую по распределению системой Радемахера на $[0,1]$. Это означает, что

$$
\mathrm{P}\left\{\omega \in \Omega:\left|\sum_{i=1}^{m} a_{i} f_{n_{i}}(\omega)\right|>z\right\} \leqslant C\left|\left\{t \in[0,1]:\left|\sum_{i=1}^{m} a_{i} r_{i}(t)\right|>\frac{z}{C}\right\}\right|
$$

где $C>0$ не зависит от $m \in \mathbb{N}, a_{i} \in \mathbb{R}(i=1, \ldots, m)$ и $z>0$.

Библиография: 16 названий.

0. Введение. Пусть $r_{i}(t)=\operatorname{sign} \sin 2^{i-1} \pi t(i=1,2, \ldots)$ - система функций Радемахера на $[0,1]$. В дальнейшем будем говорить, что система $\left\{f_{i}\right\}_{i=1}^{\infty}$ случайных величин, определенных на вероятностном пространстве $(\Omega, \Sigma, \mathrm{P})$, мажсорируется по распределению системой Радемахера, если существует $C>0$ такое, что для произвольных $m \in \mathbb{N}, a_{i} \in \mathbb{R}(i=1, \ldots, m)$ и $z>0$

$$
\mathrm{P}\left\{\omega \in \Omega:\left|\sum_{i=1}^{m} a_{i} f_{i}(\omega)\right|>z\right\} \leqslant C\left|\left\{t \in[0,1]:\left|\sum_{i=1}^{m} a_{i} r_{i}(t)\right|>\frac{z}{C}\right\}\right|
$$

(здесь и далее, $|e|$ - мера Лебега множества $e \subset[0,1]$ ).

Пусть $\left\{f_{n}\right\}_{n=1}^{\infty}-$ равномерно ограниченная ортонормированная система случайных величин на вероятностном пространстве $(\Omega, \Sigma, \mathrm{P})$. Хорошо известно (см., например, $[1$, c. 287]), что из нее можно выделить подсистему $\left\{f_{n_{i}}\right\}_{i=1}^{\infty}$, которая является $S_{p}$-системой сразу для всех $p<\infty$. Это означает, что

$$
\left\|\sum_{i=1}^{m} a_{i} f_{n_{i}}\right\|_{p} \leqslant K_{p}\left(\sum_{i=1}^{m} a_{i}^{2}\right)^{1 / 2}
$$

где $K_{p}>0$ не зависит от $m \in \mathbb{N}$ и $a_{i} \in \mathbb{R}(i=1, \ldots, m)$.

Основной результат работы является в определенном смысле усилением сформулированного утверждения. Будет доказано, что из любой равномерно ограниченной ортонормированной системы случайных величин можно выделить подсистему, которая мажорируется по распределению системой Радемахера. 
Для доказательства нам потребуется ряд утверждений, представляющих также и самостоятельньй интерес. Напомним сначала некоторые определения.

Если $X$ - случайная величина, определенная на вероятностном пространстве $(\Omega, \Sigma, \mathrm{P})$, то $n_{|X|}(z)=\mathrm{P}\{\omega \in \Omega:|X(\omega)|>z\}$, а $X^{*}(t)(t \in[0,1])$ - невозрастающая непрерьвная слева перестановка функции $|X|$. Как известно $\left[2\right.$, с. 83], $|X|$ и $X^{*}$ равноизмеримы, т.е. для всех $z>0 n_{|X|}(z)=n_{X^{*}}(z)$. Банахово пространство $E$ случайных величин $X=X(\omega)$ на $(\Omega, \Sigma, \mathrm{P})$ будем называть симметричным, если

1) из того, что $|X| \leqslant|Y|$ почти всюду и $Y \in E$, следует $X \in E$ и $\|X\| \leqslant\|Y\|$;

2) из того, что $n_{|X|}(z)=n_{|Y|}(z)(z>0)$ и $Y \in E$, следует $X \in E$ и $\|X\|=\|Y\|$.

Кроме $L_{p}$-пространств $(1 \leqslant p \leqslant \infty)$ важные примеры симметричных пространствпространство Орлича $L_{S}$ :

$$
\|x\|_{S}=\inf \left\{u>0: \int_{\Omega} S\left(\frac{|X(\omega)|}{u}\right) d P(\omega) \leqslant 1\right\}
$$

пространство Марцинкевича $M(\varphi)$ :

$$
\|x\|_{M(\varphi)}=\sup \left\{\frac{1}{\varphi(t)} \int_{0}^{t} X^{*}(s) d s: 0<t \leqslant 1\right\}
$$

и пространство Лоренца $\Lambda_{p}(\varphi)$ :

$$
\|x\|_{\varphi, p}=\left\{\int_{0}^{1}\left(X^{*}(s)\right)^{p} d \varphi(s)\right\}^{1 / p} .
$$

Здесь $S(t) \geqslant 0$ - выпуклая непрерьвная функция на $[0, \infty), \varphi(t) \geqslant 0$ - вогнутая возрастающая функция на $(0,1]$.

Важную роль в теории интерполящии операторов играет $\mathscr{K}$-функционал Петре (см., например, [3]):

$$
\mathscr{K}\left(t, x ; E_{0}, E_{1}\right)=\inf \left\{\left\|x_{0}\right\|_{E_{0}}+t\left\|x_{1}\right\|_{E_{1}}: x=x_{0}+x_{1}, x_{i} \in E_{i}\right\},
$$

где $E_{0}$ и $E_{1}$ - симметричные пространства, $x \in E_{0}+E_{1}, t>0$. Нетрудно показать, что при фиксированном $x \in E_{0}+E_{1} \mathscr{K}$-функционал-вогнутая возрастающая функция от $t[3$, c. 55$]$.

Выражение $F_{1} \asymp F_{2}$ в дальнейшем означает, что для некоторого $C>0 C^{-1} F_{2} \leqslant$ $F_{1} \leqslant C F_{2}$, причем константа $C$, как правило, не зависит от всех или части аргументов $F_{1}$ и $F_{2}$. Если $1 \leqslant p<\infty, X-$ случайная величина на $(\Omega, \Sigma, \mathrm{P})$, то

$$
\|X\|_{p}=\left\{\int_{\Omega}|X(\omega)|^{p} d P(\omega)\right\}^{1 / p}
$$

если же $a=\left(a_{i}\right)_{i=1}^{\infty}$, то

$$
\|a\|_{p}=\left\{\sum_{i=1}^{\infty}\left|a_{i}\right|^{p}\right\}^{1 / p}
$$


1. $\mathscr{K}$-функционал на суммах Радемахера. В работе [4] (см. также [5]) показано, что для функций вида

$$
x(t)=\sum_{i=1}^{\infty} a_{i} r_{i}(t), \quad a=\left(a_{i}\right)_{i=1}^{\infty} \in \ell_{2}
$$

выполнено

$$
\mathscr{K}\left(t, x ; L_{\infty}, G\right) \asymp \mathscr{K}\left(t, a ; \ell_{1}, \ell_{2}\right),
$$

где $G$ - замыкание $L_{\infty}$ в пространстве Орлича $L_{N}, N(t)=e^{t^{2}}-1$. Получим аналогичное соотношение для $\mathscr{K}$-функционала $\mathscr{K}\left(t, x ; L_{1}, L_{\infty}\right)$.

Tеорема 1. С константами, не зависящими от $a=\left(a_{i}\right)_{i=1}^{\infty} u 0<u \leqslant 1$,

$$
\mathscr{K}\left(u, \sum a_{i} r_{i} ; L_{1}, L_{\infty}\right) \asymp u \mathscr{K}\left(\ln ^{1 / 2} \frac{3}{u}, a ; \ell_{1}, \ell_{2}\right) .
$$

ДокАЗАТЕЛЬСтво. Для $a=\left(a_{i}\right)_{i=1}^{\infty} \in \ell_{2}$ положим $\varphi_{a}(t)=\mathscr{K}\left(t, a ; \ell_{1}, \ell_{2}\right)$. В работе [6] показано, что для функций вида (1)

$$
\begin{aligned}
n_{|x|}\left(\varphi_{a}(t)\right) & \leqslant 2 e^{-t^{2} / 2}, \\
n_{|x|}\left(A^{-1} \varphi_{a}(t)\right) & \geqslant A^{-1} e^{-A t^{2}},
\end{aligned}
$$

где $A>1$ не зависит от $a$ и $t>0$.

Из неравенства (3) по определению перестановки $x^{*}\left(3 e^{-t^{2} / 2}\right) \leqslant \varphi_{a}(t)$ или после замены $s=3 e^{-t^{2} / 2}$ имеет место

$$
x^{*}(s) \leqslant \sqrt{2} \varphi_{a}\left(\ln ^{1 / 2} \frac{3}{s}\right), \quad 0<s \leqslant 1 .
$$

Отсюда

$$
\int_{0}^{u} x^{*}(s) d s \leqslant \sqrt{2} \int_{0}^{u} \varphi_{a}\left(\ln ^{1 / 2} \frac{3}{s}\right) d s, \quad 0<u \leqslant 1 .
$$

С другой стороны, из неравенства (4) следует

$$
x^{*}(s) \geqslant A^{-1} \varphi_{a}\left(A^{-1 / 2} \ln ^{1 / 2}\left(A^{-1} s^{-1}\right)\right), \quad 0<s<A^{-1} .
$$

Так как функция $\varphi_{a}(u)$ вогнута, вьполнено

$$
x^{*}\left(\frac{t}{3 A}\right) \geqslant A^{-3 / 2} \varphi_{a}\left(\ln ^{1 / 2} \frac{3}{t}\right), \quad 0<t<1 .
$$

Следовательно, для всех $0<u \leqslant 1$

$$
\int_{0}^{u} x^{*}(s) d s \geqslant \int_{0}^{u /(3 A)} x^{*}(s) d s \geqslant 3^{-1} A^{-5 / 2} \int_{0}^{u} \varphi_{a}\left(\ln ^{1 / 2} \frac{3}{t}\right) d t .
$$

Таким образом, с константой, не зависящей от $a=\left(a_{i}\right)_{i=1}^{\infty}$ и $0<u \leqslant 1$,

$$
\int_{0}^{u} x^{*}(s) d s \asymp \int_{0}^{u} \varphi_{a}\left(\ln ^{1 / 2} \frac{3}{s}\right) d s .
$$


Докажем теперь, что

$$
\int_{0}^{u} \varphi_{a}\left(\ln ^{1 / 2} \frac{3}{s}\right) d s \asymp u \varphi_{a}\left(\ln ^{1 / 2} \frac{3}{u}\right) .
$$

Пусть сначала $u=3^{-k}, k \in \mathbb{N}$. Ввиду вогнутости $\varphi_{a}(t)$

$$
\begin{aligned}
\int_{0}^{3^{-k}} \varphi_{a}\left(\ln ^{1 / 2} \frac{3}{s}\right) d s & =\sum_{i=k}^{\infty} \int_{3^{-i-1}}^{3^{-i}} \varphi_{a}\left(\ln ^{1 / 2} \frac{3}{s}\right) d s \\
& \asymp \sum_{i=k}^{\infty} \varphi_{a}(\sqrt{i \ln 3}) 3^{-i} \asymp \sum_{i=k}^{\infty} \varphi_{a}(\sqrt{i}) 3^{-i}
\end{aligned}
$$

В то же время,

$$
\sum_{i=k}^{\infty} \varphi_{a}(\sqrt{i}) 3^{-i}=\sum_{j=0}^{\infty} \sum_{i=k 3^{j}}^{k 3^{j+1}-1} \varphi_{a}(\sqrt{i}) 3^{-i} \asymp \sum_{j=0}^{\infty} \varphi_{a}\left(\sqrt{k} 3^{j / 2}\right) 3^{-k 3^{j}}
$$

Так как последняя сумма больше чем $\varphi_{a}(\sqrt{k}) 3^{-k}$ и меньше чем

$$
\varphi_{a}(\sqrt{k}) 3^{-k} \sum_{j=0}^{\infty} 3^{j / 2+k-k 3^{j}} \leqslant \varphi_{a}(\sqrt{k}) 3^{-k} \sum_{j=0}^{\infty} 3^{1-j / 2} \leqslant 6 \sqrt{3} \varphi_{a}(\sqrt{k}) 3^{-k}
$$

то для $u=3^{-k}, k \in \mathbb{N}$, соотношение (6) следует из (7).

Если $u \in(0,1]$ произвольно, выберем $k \in \mathbb{N}$ так, что $3^{-k} \leqslant u \leqslant 3^{-k+1}$. Тогда ввиду вогнутости рассматриваемых здесь функций

$$
\int_{0}^{u} \varphi_{a}\left(\ln ^{1 / 2} \frac{3}{s}\right) d s \asymp \int_{0}^{3^{-k}} \varphi_{a}\left(\ln ^{1 / 2} \frac{3}{s}\right) d s \asymp 3^{-k} \varphi_{a}\left(\ln ^{1 / 2} 3^{k+1}\right) \asymp u \varphi_{a}\left(\ln ^{1 / 2} \frac{3}{u}\right),
$$

и соотношение (6) доказано.

Из (5) и (6) получаем, что

$$
\int_{0}^{u} x^{*}(s) d s \asymp u \varphi_{a}\left(\ln ^{1 / 2} \frac{3}{u}\right) .
$$

Ввиду хорошо известного равенства (см., например, [3, с. 142])

$$
\mathscr{K}\left(u, x ; L_{1}, L_{\infty}\right)=\int_{0}^{u} x^{*}(s) d s
$$

последнее соотношение эквивалентно (2), и теорема доказана.

Достаточно точную аппроксимацию функции $\varphi_{a}(t)=\mathscr{K}\left(t, a ; \ell_{1}, \ell_{2}\right)$ дает известная формула Хольмстедта [7]

$$
\mathscr{K}\left(t, a ; \ell_{1}, \ell_{2}\right) \asymp \sum_{i=1}^{\left[t^{2}\right]} a_{i}^{*}+t\left(\sum_{i=\left[t^{2}\right]+1}^{\infty}\left(a_{i}^{*}\right)^{2}\right)^{1 / 2}
$$

где $\left(a_{i}^{*}\right)$ - невозрастающая перестановка последовательности $\left(\left|a_{i}\right|\right)$, a $[z]$ - целая часть числа $z$.

Тем самым, получаем 
Следствие 1. С константами, не зависящими от $a=\left(a_{i}\right)_{i=1}^{\infty} \in \ell_{2} u 0<u \leqslant 1$,

$$
\int_{0}^{u}\left(\sum_{i=1}^{\infty} a_{i} r_{i}(t)\right)^{*} d t \asymp u\left\{\sum_{i=1}^{[\ln 3 / u]} a_{i}^{*}+\ln ^{1 / 2} \frac{3}{u}\left(\sum_{i=[\ln 3 / u]+1}^{\infty}\left(a_{i}^{*}\right)^{2}\right)^{1 / 2}\right\} .
$$

ЗАМЕчАние 1. Так как для произвольных $E_{0}, E_{1}$ и $u>0$

$$
\mathscr{K}\left(u, x ; E_{0}, E_{1}\right)=u \mathscr{K}\left(\frac{1}{u}, x ; E_{1}, E_{0}\right),
$$

из соотношения (2) следует

$$
\mathscr{K}\left(u, x ; L_{\infty}, L_{1}\right) \asymp \varphi_{a}\left(\ln ^{1 / 2} 3 u\right), \quad u \geqslant 1,
$$

или эквивалентно:

$$
\mathscr{K}\left(u, x ; L_{\infty}, L_{1}\right) \asymp \varphi_{a}\left(\ln ^{1 / 2}\left(1+u^{2}\right)\right), \quad u \geqslant 1 .
$$

Если же $0<u<1$, то из определения $\mathscr{K}$-функционала, а также из неравенств $\|x\|_{1} \leqslant\|x\|_{\infty},\|a\|_{2} \leqslant\|a\|_{1}$ и $\|x\|_{1} \leqslant\|a\|_{2} \leqslant \sqrt{2}\|x\|_{1}$ (неравенство Хинчина для $L_{1}$-нормы с константой из [8]) получаем

$$
\mathscr{K}\left(u, x ; L_{\infty}, L_{1}\right) \asymp u\|x\|_{1} \asymp u\|a\|_{2} \asymp \varphi_{a}(u) \asymp \varphi_{a}\left(\ln ^{1 / 2}\left(1+u^{2}\right)\right) .
$$

В итоге приходим к соотношению

$$
\mathscr{K}\left(u, x ; L_{\infty}, L_{1}\right) \asymp \mathscr{K}\left(\ln ^{1 / 2}\left(1+u^{2}\right), a ; \ell_{1}, \ell_{2}\right),
$$

справедливому уже при всех $u>0$.

ЗАмечАниЕ 2. Совершенно аналогично для функций вида (1) можно доказать, что

$$
\begin{aligned}
& \mathscr{K}\left(u, x ; L_{p}, L_{\infty}\right) \asymp \mathscr{K}\left(\ln ^{1 / 2} \frac{3}{u}, a ; \ell_{1}, \ell_{2}\right), \quad 0<u \leqslant 1, \\
& \mathscr{K}\left(u, x ; L_{\infty}, L_{p}\right) \asymp \mathscr{K}\left(\ln ^{1 / 2}\left(1+u^{2}\right), a ; \ell_{1}, \ell_{2}\right), \quad u>0,
\end{aligned}
$$

где $1 \leqslant p<\infty$ (константы этих эквивалентностей, конечно, зависят от $p$ ).

Отсюда получаем

СлЕДСТВИЕ 2. С константами, не зависящими от $a=\left(a_{i}\right)_{i=1}^{\infty} u 0<u \leqslant 1$,

$$
\mathscr{K}\left(u, \sum a_{i} r_{i} ; L_{p}, L_{\infty}\right) \asymp \mathscr{K}\left(u, \sum a_{i} r_{i} ; L_{1}, L_{\infty}\right) .
$$

2. О сравнении функций распределения. В дальнейшем нам потребуется вариант доказанного в работе [9] принципа эквивалентности функций распределения. Доказательство его совершенно аналогично доказательству самого принципа (см. [9]). 
ПРЕДЛОЖЕНИЕ 1. Пусть $X \geqslant 0 u Y \geqslant 0$ - две случайные величины, определенные на, вообще говоря, разных вероятностных пространствах, $\left\{X_{i}\right\}_{i=1}^{\infty},\left\{Y_{i}\right\}_{i=1}^{\infty}-$ независимые "копии" $X$ и $Y$ соответственно (т.е. $n_{X_{i}}(z)=n_{X}(z), n_{Y_{i}}(z)=$ $\left.n_{Y}(z)\right)$. Предположим, что

$$
\begin{gathered}
\left\|\max _{i=1, \ldots, n} Y_{i}\right\|_{1} \leqslant C_{1}\left\|_{i=1, \ldots, n} X_{i}\right\|_{1}, \\
\left\|\max _{i=1, \ldots, n} X_{i}\right\|_{2} \leqslant C_{2}\left\|_{i=1, \ldots, n} X_{i}\right\|_{1},
\end{gathered}
$$

где $C_{1}>0$ и $C_{2}>0$ не зависят от $n \in \mathbb{N}$.

Тогда найдется $C>0$, зависящее только от $C_{1} u C_{2}$, такое, ито при всех $z>0$

$$
n_{Y}(z) \leqslant C n_{X}\left(\frac{z}{C}\right)
$$

Покажем, что условия (10) и (11) можно сформулировать, используя понятие $\mathscr{K}$-функционала.

ПРЕДЛОЖЕНИЕ 2. Предположим, что $\left\{X_{i}\right\}_{i=1}^{\infty}-$ последовательность независимых "копий" случайной величины $X \geqslant 0$ на вероятностном пространстве $(\Omega, \Sigma, \mathrm{P})$, $1 \leqslant p<\infty$.

Тогда для любого $n \in \mathbb{N}$

$$
\left\|\max _{i=1, \ldots, n} X_{i}\right\|_{p} \asymp n \mathscr{K}\left(\frac{1}{n}, X^{*} ; L_{p}[0,1], L_{\infty}[0,1]\right)
$$

с константой, не зависящей от $X$ и $n$.

ДокаЗАтЕльство. Так как $X_{i}(i=1,2, \ldots, n)$ независимы, для $\bar{X}=\max _{i=1, \ldots, n} X_{i}$

$$
\begin{aligned}
\|\bar{X}\|_{p}^{p} & =\int_{\Omega} \cdots \int_{\Omega} \max \left\{X^{p}\left(\omega_{1}\right), \ldots, X^{p}\left(\omega_{n}\right)\right\} d P\left(\omega_{1}\right) \cdots d P\left(\omega_{n}\right) \\
& =n \int \cdots \int_{\left\{X\left(\omega_{i}\right) \leqslant X\left(\omega_{1}\right)\right\}} X^{p}\left(\omega_{1}\right) d P\left(\omega_{n}\right) \cdots d P\left(\omega_{2}\right) d P\left(\omega_{1}\right) \\
& =n \int_{\Omega} X^{p}\left(\omega_{1}\right)\left(\mathrm{P}\left\{\omega: X(\omega) \leqslant X\left(\omega_{1}\right)\right\}\right)^{n-1} d P\left(\omega_{1}\right) \\
& =n \int_{\Omega} X^{p}\left(\omega_{1}\right)\left(1-n_{X}\left(X\left(\omega_{1}\right)\right)\right)^{n-1} d P\left(\omega_{1}\right) .
\end{aligned}
$$

Ввиду равноизмеримости функций $X\left(\omega_{1}\right)\left(\omega_{1} \in \Omega\right)$ и $X^{*}(t)(t \in[0,1])$, а также того, что $n_{X}\left(X^{*}(t)\right)=t$ для почти всех $t \in[0,1][2$, c. 83], отсюда получим

$$
\|\bar{X}\|_{p}^{p}=n \int_{0}^{1}\left(X^{*}\right)^{p}(t)\left(1-n_{X}\left(X^{*}(t)\right)\right)^{n-1} d t=n \int_{0}^{1}\left(X^{*}\right)^{p}(t)(1-t)^{n-1} d t .
$$

Из последнего равенства следует

$$
\|\bar{X}\|_{p}=\left\|X^{*}\right\|_{\Lambda_{p}\left(\varphi_{n}\right)},
$$


где $\Lambda_{p}\left(\varphi_{n}\right)$ - симметричное пространство Лоренца, построенное по функции $\varphi_{n}(t)=$ $1-(1-t)^{n}$.

Покажем, что для любых $n \in \mathbb{N}$ и $t \in[0,1]$

$$
e^{-1} \psi_{n}(t) \leqslant \varphi_{n}(t) \leqslant \psi_{n}(t)
$$

где $\psi_{n}(t)=\min \{1, n t\}$.

Так как $\varphi_{1}(t)=\psi_{1}(t)=1$, можно считать, что $n \geqslant 2$. Если $t \geqslant 1 / n$, то $\varphi_{n}(t) \leqslant 1=$ $\psi_{n}(t)$, а $\varphi_{n}(t) \geqslant \varphi_{n}(1 / n)=1-(1-1 / n)^{n} \geqslant 1-e^{-1} \geqslant \psi_{n}(t) / e$ (последовательность $\left.(1-1 / n)^{n} \uparrow 1 / e\right)$. В случае $0<t \leqslant 1 / n$ рассмотрим функцию

$$
f_{n}(t)=\psi_{n}(t)-\varphi_{n}(t)=n t-1+(1-t)^{n} .
$$

Так как $f_{n}(0)=0$ и $f_{n}^{\prime}(t)=n-n(1-t)^{n-1} \geqslant 0$, то $f_{n}(t) \geqslant 0$, что эквивалентно правой части неравенства (13) в случае $0 \leqslant t \leqslant 1 / n$. В то же время, для функции

$$
g_{n}(t)=\frac{\psi_{n}(t)}{e}-\varphi_{n}(t)=\frac{n t}{e}-1+(1-t)^{n}
$$

выполнено

$g_{n}(0)=0, \quad g_{n}^{\prime}(t)=\frac{n}{e}-n(1-t)^{n-1}=n\left(\frac{1}{e}-(1-t)^{n-1}\right) \leqslant n\left(\frac{1}{e}-\left(1-\frac{1}{n}\right)^{n-1}\right) \leqslant 0$ (последовательность $\left.(1-1 / n)^{n-1} \downarrow 1 / e\right)$. Следовательно, $g_{n}(t) \leqslant 0$, что эквивалентно левой части (13) при $0 \leqslant t \leqslant 1 / n$, и доказательство этого соотношения закончено.

Из (13) следует, что для произвольной измеримой на $[0,1]$ функции $x(t)$

$$
\frac{1}{e}\|x\|_{\Lambda\left(\psi_{n}\right)} \leqslant\|x\|_{\Lambda\left(\varphi_{n}\right)} \leqslant\|x\|_{\Lambda\left(\psi_{n}\right)} .
$$

Значит, ввиду (12), а также формулы для $\mathscr{K}$-функционала [3, с. 142] выполнено

$$
\|\bar{X}\|_{p} \asymp\left\|X^{*}\right\|_{\Lambda_{p}\left(\psi_{n}\right)}=n\left\{\int_{0}^{1 / n}\left(X^{*}(t)\right)^{p} d t\right\}^{1 / p} \asymp n \mathscr{K}\left(\frac{1}{n}, X^{*} ; L_{p}, L_{\infty}\right)
$$

с константой, не зависящей от $X$ и $n$.

Следствием предложений 1 и 2 является

ТЕОрема 2. Пусть $X \geqslant 0 u Y \geqslant 0$ - две случайные величины, определенные на, вообще говоря, разных вероятностных пространствах. Предполоэмим, что

$$
\begin{gathered}
\int_{0}^{t} Y^{*}(s) d s \leqslant C_{1} \int_{0}^{t} X^{*}(s) d s, \quad, 0<t \leqslant 1 \\
\int_{0}^{t}\left(X^{*}(s)\right)^{2} d s \leqslant C_{2}\left(\int_{0}^{t} X^{*}(s) d s\right)^{2}, \quad 0<t \leqslant 1 .
\end{gathered}
$$

Тогда существует $C>0$, зависящее лишь от $C_{1}$ и $C_{2}$, такое, что для всех $z>0$

$$
n_{Y}(z) \leqslant C n_{X}\left(\frac{z}{C}\right) .
$$


СлЕДСТВИЕ 3. Пусть Е - симметричное пространство на $[0,1], x=x(t) u$ $y=y(t)$ - измеримые на $[0,1]$ функиии, для которых выполнены условия (14) $u(15)$. Тогда если $x \in E$, то $y \in E u\|y\|_{E} \leqslant C\|x\|_{E}$, где $C>0$ зависит лишь от $C_{1}$ и $C_{2}$.

ДоКАЗАТЕЛЬСТво. Воспользовавшись следствием 2, получим, что при всех $z>0$

$$
n_{|y|}(z) \leqslant C^{\prime} n_{|x|}\left(\frac{z}{C^{\prime}}\right)
$$

или эквивалентно: $y^{*}(t) \leqslant C^{\prime} \sigma_{C^{\prime}} x^{*}(t)(t \in(0,1])$, где $\sigma_{\tau} x(t)=x(t / \tau)$ - оператор растяжения. Так как $\sigma_{\tau}$ ограничен в любом симметричном пространстве и $\left\|\sigma_{\tau}\right\|_{E \rightarrow E} \leqslant$ $\max \{1, \tau\}\left[2\right.$, c. 133], отсюда следует, что $\|y\|_{E} \leqslant C\|x\|_{E}$, где $C=C^{\prime} \max \left\{1, C^{\prime}\right\}$.

ЗАмЕчанИЕ 3 . Легко убедиться в том, что условие (15) в следствии 3 существенно. Более того, если $E$ - симметричное пространство, не интерполяционное между пространствами $L_{1}$ и $L_{\infty}$, то всегда можно найти функцию $x \in E$ и линейный оператор $T$, ограниченно действующий в $L_{1}$ и $L_{\infty}$, такие, что $y=T x \notin E[2$, c. 130,166$]$.

Применим теперь теорему 2 в случае, когда

$$
X(t)=\left|\sum_{i=1}^{m} a_{i} r_{i}(t)\right|, \quad Y(\omega)=\left|\sum_{i=1}^{m} a_{i} f_{i}(\omega)\right|, \quad m \in \mathbb{N}, \quad a_{i} \in \mathbb{R}
$$

где $r_{i}(t)$ - функции Радемахера на $[0,1]$, а $f_{i}(\omega)$ - случайные величины на некотором вероятностном пространстве $(\Omega, \Sigma, \mathrm{P})$.

ТЕОРемА 3. Пусть $\left\{f_{i}\right\}_{i=1}^{\infty}-$ ортонормированная система случайных величин на вероятностном пространстве $(\Omega, \Sigma, \mathrm{P}),\left|f_{i}(\omega)\right| \leqslant M, \omega \in \Omega, i=1,2, \ldots$.

Следуюшие условия әквивалентны:

1) для произвольной последовательности $a=\left(a_{i}\right)_{i=1}^{\infty} \in \ell_{2}$ функиия

$$
f=\sum_{i=1}^{\infty} a_{i} f_{i} \in L_{N}
$$

əде $N(t)=e^{t^{2}}-1$

2) существует $C>0$, не зависящее от $m \in \mathbb{N}, a=\left(a_{i}\right)_{i=1}^{m} u t \in[0,1]$, такое, чтоo

$$
\int_{0}^{t}\left(\sum_{i=1}^{m} a_{i} f_{i}\right)^{*}(s) d s \leqslant C_{1} t \mathscr{K}\left(\ln ^{1 / 2} \frac{3}{t}, a ; \ell_{1}, \ell_{2}\right) ;
$$

3) система $\left\{f_{i}\right\}_{i=1}^{\infty}$ махсорируется по распределению системой Радемахера.

ДоказАтЕльство. Сначала докажем импликацию 1) $\Longrightarrow 2$ ). Как известно [10], пространство Орлича $L_{N}$ совпадает с пространством Марцинкевича $M(\varphi)$, где $\varphi(t)=$ $t \log _{2}^{1 / 2}(2 / t)$. Следовательно, по определению нормы в $M(\varphi)$

$$
\int_{0}^{t}\left(\sum_{i=1}^{\infty} a_{i} f_{i}\right)^{*}(s) d s \leqslant C_{1} t \log _{2}^{1 / 2} \frac{2}{t}\|a\|_{2}
$$

где $C_{1}$ не зависит от $a=\left(a_{i}\right)_{i=1}^{\infty} \in \ell_{2}$ и $t \in[0,1]$. 
Пусть $a_{k}^{*}=\left|a_{i_{k}}\right|(k=1,2, \ldots)$ - невозрастающая перестановка последовательности $\left(\left|a_{i}\right|\right)_{i=1}^{\infty}$. Ввиду неравенства (17), а также условий теоремы для произвольного $j \in \mathbb{N}$

$$
\begin{aligned}
\int_{0}^{2^{-j}}\left(\sum_{i=1}^{\infty} a_{i} f_{i}\right)^{*}(s) d s & \leqslant \int_{0}^{2^{-j}}\left(\sum_{k=1}^{j} a_{i_{k}} f_{i_{k}}\right)^{*}(s) d s+\int_{0}^{2^{-j}}\left(\sum_{k=j+1}^{\infty} a_{i_{k}} f_{i_{k}}\right)^{*}(s) d s \\
& \leqslant 2^{-j} M \sum_{k=1}^{j}\left|a_{i_{k}}\right|+C_{1} 2^{-j} \log _{2}^{1 / 2}\left(2^{j+1}\right)\left(\sum_{k=j+1}^{\infty}\left|a_{i_{k}}\right|^{2}\right)^{1 / 2} \\
& \leqslant 2^{-j} \max \left\{M, 2 C_{1}\right\}\left(\sum_{k=1}^{j} a_{k}^{*}+\sqrt{j}\left(\sum_{k=j+1}^{\infty}\left(a_{k}^{*}\right)^{2}\right)^{1 / 2}\right) \\
& \leqslant 2^{-j} C_{2} \max \left\{M, 2 C_{1}\right\} \mathscr{K}\left(\sqrt{j}, a ; \ell_{1}, \ell_{2}\right)
\end{aligned}
$$

где последнее неравенство следует из формулы Хольмстедта (8).

Таким образом, соотношение (16) вьполнено для $t=2^{-j}(j \in \mathbb{N})$. Так как рассматриваемые здесь функции вогнуты, оно справедливо и при всех $t \in[0,1]$.

Импликация 2 ) $\Longrightarrow 3$ ) следует из теоремы 1 , следствия 2 и теоремы 2 .

Предположим, наконец, что выполнено условие 3 ). Хорошо известно (см., например, $\left[11\right.$, с. 342]), что функция $g=\sum_{i=1}^{\infty} a_{i} r_{i} \in L_{N}$, если $a=\left(a_{i}\right)_{i=1}^{\infty} \in \ell_{2}$. По условию для функции $f=\sum_{i=1}^{\infty} a_{i} f_{i}$ вьполнено

$$
n_{|f|}(z) \leqslant C n_{|g|}\left(\frac{z}{C}\right)
$$

Следовательно, ввиду симметричности пространства $L_{N}$, как и при доказательстве следствия 3 , получаем, что $f \in L_{N}$, и теорема доказана.

Далее нам потребуются следующие обозначения (подробнее см. в $[12$, гл. 1]). Пусть $(\Omega, \Sigma, \mathrm{P})$ - вероятностноепространство, $\Re-\sigma$-подалгебра $\sigma$-алгебры $\Sigma$. Если $f: \Omega \rightarrow \mathbb{R}-$ случайная величина, то $\mathrm{E}[f \mid \mathfrak{R}]$ - условное математическое ожидание $f$ относительно $\Re$. Если $\mathfrak{R}-\sigma$-подалгебра, порожденная случайньпи величинами $g_{1}, g_{2}, \ldots, g_{n}$, то условноематематическое ожидание относительно нее обозначим через $\mathrm{E}\left[f \mid g_{1}, \ldots, g_{n}\right]$. В частности,

$$
\mathrm{E}[f]=\int_{\Omega} f(\omega) d P(\omega) .
$$

Напомним, что последовательность $\left\{f_{n}\right\}_{n=1}^{\infty}$ случайных величин на вероятностном пространстве $(\Omega, \Sigma, \mathrm{P})$ называется мультипликативной, если для любых $k \in \mathbb{N}$ и $n_{1}<$ $n_{2}<\cdots<n_{k}$

$$
\mathrm{E}\left[f_{n_{1}} f_{n_{2}} \cdots f_{n_{k}}\right]=0 .
$$

По теореме 3 и следствию 3 из работы [13] получаем

СлЕДСТВИЕ 4. Если $\left\{f_{i}\right\}_{i=1}^{\infty}-$ равномерно ограниченная мультипликативнвя ортонормированная система на $(\Omega, \Sigma, \mathrm{P})$, то она махорируетсл по распределению системой Радемахера. 
ЗАмечАниЕ 4. В [13] показано, что в условиях следствия 4 вектор $\left(f_{1}, f_{2}, \ldots, f_{n}\right)$ имеет одинаковое распределение с условным математическим ожиданием $\mathrm{E}\left[\left(g_{1}, g_{2}, \ldots, g_{n}\right) \mid \mathfrak{R}\right]$ вектора $\left(g_{1}, g_{2}, \ldots, g_{n}\right)$, равнораспределенного с вектором $\left(r_{1}, r_{2}, \ldots, r_{n}\right)$. Непосредственно отсюда утверждение следствия 4 не вытекает, так как оператор условного математического ожидания может быть даже не ограничен в симметричном пространстве, если оно не интерполяционно между $L_{1}$ и $L_{\infty}$ (см., например, [14, с. 128-129]).

3. Выделение экспоненциально интегрируемых подсистем. В доказательстве следующего утверждения используется идея из работы $[15$, лемма A].

ЛЕмма. Пусть $\left\{f_{n}\right\}_{n=1}^{\infty}-$ последовательность случайных величин, определенных на вероятностном пространстве $(\Omega, \Sigma, \mathrm{P}),\left|f_{n}(\omega)\right| \leqslant M, \omega \in \Omega, n \in \mathbb{N} u$ $f_{n} \rightarrow 0$ слабо в $L_{2}(\Omega)$. Тогда существуют $\left\{f_{n_{i}}\right\}_{i=1}^{\infty} \subset\left\{f_{n}\right\}_{n=1}^{\infty}$ и последовательность случайных величин $\left\{g_{k}\right\}_{k=1}^{\infty}$ такие, что

1) $\left|g_{k}(\omega)\right| \leqslant 2 M, \omega \in \Omega, k \in \mathbb{N}$

2) $\mathrm{E}\left[g_{k} \mid g_{1}, \ldots, g_{k-1}\right]=0$ почти всюду на $\Omega$;

3) $\left\|f_{n_{k}}-g_{k}\right\|_{k} \leqslant 2^{-k}, k \in \mathbb{N}$.

ДоказАТЕЛьство. Воспользуемся индукцией. Так как $f_{n} \rightarrow 0$ слабо в $L_{2}(\Omega)$, можно найти $n_{1}$ такое, что

$$
\left|\mathrm{E}\left[f_{n_{1}}\right]\right| \leqslant \frac{1}{6}
$$

Существует конечнозначная функция $h_{1}$, для которой $\left|h_{1}(\omega)\right| \leqslant M$ и

$$
\mathrm{E}\left[\left|f_{n_{1}}-h_{1}\right|\right] \leqslant \frac{1}{6}
$$

Положим $g_{1}=h_{1}-\mathrm{E}\left[h_{1}\right]$. Тогда $\mathrm{E}\left[g_{1}\right]=0$ и $\left|g_{1}(\omega)\right| \leqslant 2 M$. Кроме того, из неравенств (18) и (19) следует

$$
\begin{aligned}
\left\|f_{n_{1}}-g_{1}\right\|_{1} & \leqslant \mathrm{E}\left[\left|f_{n_{1}}-h_{1}\right|\right]+\mathrm{E}\left[\left|h_{1}-g_{1}\right|\right]=\mathrm{E}\left[\left|f_{n_{1}}-h_{1}\right|\right]+\left|\mathrm{E}\left[h_{1}\right]\right| \\
& \leqslant 2 \mathrm{E}\left[\left|f_{n_{1}}-h_{1}\right|\right]+\left|\mathrm{E}\left[f_{n_{1}}\right]\right| \leqslant \frac{1}{2} .
\end{aligned}
$$

Предположим, что $k>1$ и найдены величины $f_{n_{1}}, \ldots, f_{n_{k-1}}, 1 \leqslant n_{1}<\cdots<n_{k-1}$ и $g_{1}, \ldots, g_{k-1}$ такие, что при всех $s=1, \ldots, k-1$

a) $\left|g_{s}(\omega)\right| \leqslant 2 M$

б) $g_{s}$ постоянна на множествах $e_{i}^{(s)} \subset \Omega, i=1, \ldots, i_{s}, e_{i}^{(s)} \cap e_{j}^{(s)}=\varnothing(i \neq j)$ и $\bigcup_{i} e_{i}^{(s)}=\Omega$, причем $e_{i}^{(s)}$ целиком лежат во множествах постоянства $e_{i}^{(s-1)}$, $i=1, \ldots, i_{s-1}$, функции $g_{s-1}$;

в) $\mathrm{E}\left[g_{s} \mid g_{1}, \ldots, g_{s-1}\right]=0$ почти всюду на $\Omega$;

г) $\left\|f_{n_{s}}-g_{s}\right\|_{s} \leqslant 2^{-s}$.

Найдем следующую пару величин $f_{n_{k}}$ и $g_{k}$, удовлетворяющую аналогичным условиям. Так как $f_{n} \rightarrow 0$ слабо в $L_{2}(\Omega)$, существует $n_{k}>n_{k-1}$ такое, что для всех $i=1, \ldots, i_{k-1}$

$$
\left|\int_{e_{i}^{(k-1)}} f_{n_{k}}(\omega) d P(\omega)\right| \leqslant \frac{2^{-k}}{3} \mathrm{P}\left(e_{i}^{(k-1)}\right) .
$$


Пусть $h_{k}$ - конечнозначная функция, $\left|h_{k}(\omega)\right| \leqslant M$, все множества постоянства которой целиком содержатся во множествах $e_{i}^{(k-1)}\left(i=1, \ldots, i_{k-1}\right)$ и, кроме того, для всех $i=1, \ldots, i_{k-1}$

$$
\int_{e_{i}^{(k-1)}}\left|f_{n_{k}}(\omega)-h_{k}(\omega)\right|^{k} d P(\omega) \leqslant\left(\frac{2^{-k}}{3}\right)^{k} \mathrm{P}\left(e_{i}^{(k-1)}\right) .
$$

Положим $g_{k}=h_{k}-\mathrm{E}\left[h_{k} \mid g_{1}, \ldots, g_{k-1}\right]$. Тогда $\mathrm{E}\left[g_{k} \mid g_{1}, \ldots, g_{k-1}\right]=0$ почти всюду на $\Omega$ и $\left|g_{k}(\omega)\right| \leqslant 2 M$. Так как для $\omega \in e_{i}^{(k-1)}$ и произвольной случайной величины $f$ на $\Omega$

$$
\mathrm{E}\left[f \mid g_{1}, \ldots, g_{k-1}\right](\omega)=\mathrm{P}\left(e_{i}^{(k-1)}\right)^{-1} \int_{e_{i}^{(k-1)}} f(u) d P(u)
$$

то ввиду (21)

$$
\begin{gathered}
\left|\mathrm{E}\left[f_{n_{k}}-h_{k} \mid g_{1}, \ldots, g_{k-1}\right]\right| \leqslant \max _{i=1, \ldots, i_{k-1}} \mathrm{P}\left(e_{i}^{(k-1)}\right)^{-1} \int_{e_{i}^{(k-1)}}\left|f_{n_{k}}(u)-h_{k}(u)\right| d P(u) \\
\leqslant \max _{i=1, \ldots, i_{k-1}} \mathrm{P}\left(e_{i}^{(k-1)}\right)^{-1 / k}\left(\int_{e_{i}^{(k-1)}}\left|f_{n_{k}}(u)-h_{k}(u)\right|^{k} d P(u)\right)^{1 / k} \leqslant \frac{2^{-k}}{3} .
\end{gathered}
$$

Поэтому, используя (20), получим

$$
\left|\mathrm{E}\left[h_{k} \mid g_{1}, \ldots, g_{k-1}\right]\right| \leqslant\left|\mathrm{E}\left[f_{n_{k}}-h_{k} \mid g_{1}, \ldots, g_{k-1}\right]\right|+\left|\mathrm{E}\left[f_{n_{k}} \mid g_{1}, \ldots, g_{k-1}\right]\right| \leqslant \frac{2^{-k+1}}{3} .
$$

Отсюда и из неравенств (21) следует

$$
\left\|f_{n_{k}}-g_{k}\right\|_{k} \leqslant\left\|f_{n_{k}}-h_{k}\right\|_{k}+\left\|h_{k}-g_{k}\right\|_{k} \leqslant \frac{2^{-k}}{3}+\left\|\mathrm{E}\left[h_{k} \mid g_{1}, \ldots, g_{k-1}\right]\right\|_{k} \leqslant 2^{-k}
$$

и доказательство леммы закончено.

Следующее утверж дение без доказательства сформулировано в работе В. Ф. Гапошкина [16, теорема 1.3.2]. Доказательство сообщено мне автором и приведено здесь с его разрешения.

ТЕОРемА 4. Пусть $\left\{f_{n}\right\}_{n=1}^{\infty}$ - ортонормированная последовательность случайных величин на вероятностном пространстве $(\Omega, \Sigma, \mathrm{P}),\left|f_{n}(\omega)\right| \leqslant M, \omega \in \Omega$, $n \in \mathbb{N}$. Существует подпоследовательность $\left\{f_{n_{i}}\right\}_{i=1}^{\infty} \subset\left\{f_{n}\right\}_{n=1}^{\infty}$ такая, что для любой последовательности $a=\left(a_{i}\right)_{i=1}^{\infty} \in \ell_{2}$ функиия $f=\sum_{i=1}^{\infty} a_{i} f_{n_{i}} \in L_{N}$, әде $L_{N}$ - пространство Орлича, построенное по функиии $N(t)=e^{t^{2}}-1$.

ДокАЗАТЕЛЬСтво. Так как $f_{n} \rightarrow 0$ слабо в $L_{2}(\Omega)$, согласно лемме существуют $\left\{f_{n_{i}}\right\}_{i=1}^{\infty} \subset\left\{f_{n}\right\}_{n=1}^{\infty}$ и $\left\{g_{k}\right\}_{k=1}^{\infty}$ такие, что $\left|g_{k}(\omega)\right| \leqslant 2 M, \mathrm{E}\left[g_{k} \mid g_{1}, \ldots, g_{k-1}\right]=0$ почти всюду и

$$
\left\|g_{k}-f_{n_{k}}\right\|_{k} \leqslant 2^{-k}, \quad k \in \mathbb{N} .
$$

Заметим, что последоватеьность $\left\{g_{k}\right\}$ мультипликативна. Действительно, так как (см. [12, с. 13]) для произвольных $k_{1}<\cdots<k_{m}$

$$
\mathrm{E}\left[g_{k_{m}} g_{k_{1}} \cdots g_{k_{m-1}} \mid g_{k_{1}}, \ldots, g_{k_{m-1}}\right]=g_{k_{1}} \cdots g_{k_{m-1}} \mathrm{E}\left[g_{k_{m}} \mid g_{k_{1}}, \ldots, g_{k_{m-1}}\right]=0
$$


то

$$
\mathrm{E}\left[g_{k_{1}} \cdots g_{k_{m-1}} g_{k_{m}}\right]=\int_{\Omega} \mathrm{E}\left[g_{k_{m}} g_{k_{1}} \cdots g_{k_{m-1}} \mid g_{k_{1}} \cdots g_{k_{m-1}}\right] d P(\omega)=0 .
$$

Ввиду [13, следствие 3] для мультипликативных систем

$$
\left\|\sum_{k=1}^{\infty} b_{k} g_{k}\right\|_{p} \leqslant C \sqrt{p}\left\|\left(b_{k}\right)\right\|_{2}
$$

где $C=C(M)>0$ не зависит от $\left(b_{k}\right) \in \ell_{2}$ и $p \in(1, \infty)$. Поэтому, используя $(22)$, для $f=\sum_{i=1}^{\infty} a_{i} f_{n_{i}}$ и произвольного $k \in \mathbb{N}$ получим

$$
\begin{aligned}
\|f\|_{k} \leqslant & \left\|\sum_{i=1}^{k} a_{i} f_{n_{i}}\right\|_{k}+\left\|\sum_{i=k+1}^{\infty} a_{i}\left(f_{n_{i}}-g_{i}\right)\right\|_{k}+\left\|\sum_{i=k+1}^{\infty} a_{i} g_{i}\right\|_{k} \\
\leqslant & \left(\sum_{i=1}^{k} a_{i}^{2}\right)^{1 / 2}\left\|\left(\sum_{i=1}^{k} f_{n_{i}}^{2}\right)^{1 / 2}\right\|_{k} \\
& +\left(\sum_{i=k+1}^{\infty} a_{i}^{2}\right)^{1 / 2}\left\|\left(\sum_{i=k+1}^{\infty}\left(f_{n_{i}}-g_{i}\right)^{2}\right)^{1 / 2}\right\|_{k}+\left\|\sum_{i=k+1}^{\infty} a_{i} g_{i}\right\|_{k} \\
\leqslant & M \sqrt{k}\left(\sum_{i=1}^{k} a_{i}^{2}\right)^{1 / 2}+\left(\sum_{i=k+1}^{\infty} a_{i}^{2}\right)^{1 / 2} \sum_{i=k+1}^{\infty}\left\|f_{n_{i}}-g_{i}\right\|_{i}+C \sqrt{k}\left(\sum_{i=k+1}^{\infty} a_{i}^{2}\right)^{1 / 2} \\
\leqslant & (C+M+1) \sqrt{k}\|a\|_{2} .
\end{aligned}
$$

Из разложения

$$
e^{u z^{2}}=\sum_{k=0}^{\infty} \frac{u^{k}}{k !} z^{2 k}, \quad u>0
$$

следует

$$
\int_{\Omega}\left(e^{u|f(\omega)|^{2}}-1\right) d P(\omega)=\sum_{k=1}^{\infty} \frac{u^{k}}{k !} \int_{\Omega}|f(\omega)|^{2 k} d P(\omega) \leqslant \sum_{k=1}^{\infty}(C+M+1)^{2 k} \frac{u^{k}}{k !}(2 k)^{k}\|a\|_{2}^{2 k} .
$$

Так как полученњый ряд сходится при $0<u<(2 e)^{-1}(C+M+1)^{-2}\left(\|a\|_{2}\right)^{-2}$, то $f$ принадлежит пространству Орлича $L_{N}, N(t)=e^{t^{2}}-1$, и теорема доказана.

Из теорем 3 и 4 следует основной результат статьи.

ТЕОРемА 5. Пусть $\left\{f_{n}\right\}_{n=1}^{\infty}-$ ортонормированная последовательность случайных величин на вероятностном пространстве $(\Omega, \Sigma, \mathrm{P}),\left|f_{n}(\omega)\right| \leqslant M, \omega \in \Omega$, $n \in \mathbb{N}$.

Тогда из $\left\{f_{n}\right\}_{n=1}^{\infty}$ можнно выделить подпоследовательность $\left\{f_{n_{i}}\right\}_{i=1}^{\infty} \subset\left\{f_{n}\right\}_{n=1}^{\infty}$, которая махорируется по распределению системой Радемахера. Последнее означает, что для некоторого $C=C(M)>0$, не зависящего от $m \in \mathbb{N}, a=\left(a_{i}\right)_{i=1}^{m} u$ $z>0$, выполнено

$$
\mathrm{P}\left\{\omega \in \Omega:\left|\sum_{i=1}^{m} a_{i} f_{n_{i}}(\omega)\right|>z\right\} \leqslant C\left|\left\{t \in[0,1]:\left|\sum_{i=1}^{m} a_{i} r_{i}(t)\right|>\frac{z}{C}\right\}\right| .
$$

В заключение автор выражает искреннюю признательность В. Ф. Гапошкину за консультации по вопросам, связанньм с последней частью работы. 


\section{СПИСОК ЦИТИРОВАННОЙ ЛИТЕРАТУРЫ}

[1] Качмаж С., Штейнгауз Г. Теория ортогональных рядов. М.: Физматгиз, 1958.

[2] Крейн С. Г., Петунин Ю.И., Семёнов Е. М. Интерполяция линейных операторов. М.: Наука, 1978.

[3] Берг $\breve{И} .$, Л ефстрем Й. Интерполяционные пространства. Введение. М.: Мир, 1980.

[4] Асташкин С. В. Об интерполяции подпространств симметричных пространств, порожденных системой Радемахера // Изв. РАЕН. Сер. МММИУ. 1997. Т. 1. №1. С. 18-35.

[5] Асташкин С. В. О рядах по системе Радемахера, "близких" к $L_{\infty} / /$ Функцион. анализ и его прилож. (в печати).

[6] Montgomery-Smith S. The distribution of Rademacher sums // Proc. Amer. Math. Soc. 1990. V. 109. № 2. P. 517-522.

[7] Holmstedt T. Interpolation of quasi-normed spaces // Math. Scand. 1970. V. 26. P. 177-199.

[8] Szarek S. J. On the best constants in the Khinchine inequality // Studia Math. 1976. V. 58. P. 197-208.

[9] Asmar N. H., Montgomery-Smith S. On the distribution of Sidon series // Ark. Mat. 1993. V. 31. № 1. P. 13-26.

[10] Рутицкий Я. Б. О некоторых классах измеримых функций // УМН. 1965. Т. 20. №4. C. $205-208$.

[11] Зигмунд А. Тригонометрические ряды. Т. 1. М.: Мир, 1965.

[12] Эллиотт Р. Стохастический анализ и его приложения. М.: Мир, 1986.

[13] Jakubowski J., Kwapien S. On multiplicative systems of functions // Bull. Acad. Polon. Sci. Ser. Sci. Math. 1979. V. 27. № 9. P. 689-694.

[14] Lindenstrauss J., Tzafriri L. Classical Banach Spaces. V. 2. Berlin: Springer, 1979.

[15] Гапошкин В. Ф. Сходимость и предельные теоремы для подпоследовательностей случайных величин // Теор. вероятн. и ее применения. 1972. Т. 17. № 3. С. 401-423.

[16] Гапошкин В.Ф. Лакунарные ряды и независимые функции // УМН. 1966. Т. 21. №6. C. $3-82$.

Самарский государственный университет 